\title{
Case Reports Covering Diagnostic, Pathogenic and Therapeutic Aspects of Diseases
}

\section{Dieudonne Kaimbo Wa Kaimbo*}

Ophthalmology and Biophysics, University of Kinshasa, Republic of Congo

In the volume 3 issue 11, Meena [1] from India, reports on a rare case of lipoma of pancreas from a 60 year-old woman who presented in emergency department with abdominal pain. Anabdominal Computer Tomography imaging after an abdominal ultrasonography examination revealed the characteristics of the lesion. Authors assert that lipoma of pancreas which represents less than $1 \%$ of all pancreatic tumours must be distinguished radiologically from replacement or infiltration fatty disorder, replacement focal fat, lipomatosis or adipose dysplasia, pseudohypertrophyclipomatosis, pancreatic teratoma, andliposarcoma.

Rozin and Toledano [2] from Israel describe a case of 29-year-old man, with 10-year history of psoriasis and sinusitis, who presented with ocular involvement. After a workup and ancillary exams, the authors believed that it is an unknown autoimmune syndrome that they called Psoriasis Periophthalmitis and Pansinusitis (PPP)-syndrome. After treatment with topical antibiotic and steroid agent during six months the affection was completely resolved. Active sinusitis with staphylococcus microbial flora as the probable pathogen was proposed to be a trigger of immune inflammation.

Shigefuku et al. [3] from Japan, discuss the relationship between nonalcoholic steatohepatitis (NASH) and sleep apnea syndrome (SAS) in reporting a case of a 35-year-old man, with a history of obesity, hyperuricemia, hypercholesteromia and liver dysfunction since youth. On the basis of clinical and laboratory findings, a diagnosis of NASH was made. Continuous positive airway pressure (CPAP), weight loss and strict diet therapy improved serum alamine aminotransferase (ALT). Authors suggested that SAS might partially contribute to the pathogenesis of NASH.

Grover et al. [4] from India, report a case report of a 63-yearold female, with a past history of Diabetes Mellitus, hypertension, hypothyroidism, who presented with recurrent depressive disorder, highth the importance of monitoring of serum sodium levels before starting antidepressant drugs. Their patient showed hyponatremia before and under Sertraline ${ }^{\circledR}$ orMilnacipran $^{\circledR}$. Fludrocortisone 50 micrgram/day associated with Sertraline ${ }^{\circledR}$ and after Milnacipran ${ }^{\bullet}$ normalized serum sodium levels.

Kenneth et al. [5] from Kazakhstan, describe a case of 54-year-old woman who was diagnosed with inflammatory-edematous forms of breast cancer stage IV metastasized to multiple organs. A combinational therapy consisting of immunocorrective and metabolism regulating therapy, as well as antimicrobial and standard chemotherapy, was shown to extend patient's life for almost two additional years. Therefore, the authors state that personalized treatment should become a main approach to treat cancer patients.

Docx et al. [6] from Belgium, present a case of 14-year-old girl who presented with food refusal, loss appetite, chronic fatigue and depression; she also developed water and sodium imbalance following ingestion of Desmopression for enuresianocturna and Carbamazepine for epileptic insult. Her past medical history also included hemolyticuremic syndrome at the age of 4.5 years. Central adrenal insufficiency was diagnosed and supplementation with hydrocortisone improved symptoms.

Saber et al. [7] from Kingdom of Saudi Arabia, report a case of a 28-year-old male who sustained a razor wound to the neck resulting in a penetrating laryngeal injury with multiple disruptions between cricoid and thyroid cartilages. Emergent and surgical management are described.

\section{References}

1. Meena BK (2013) Rare Case of Pancreatic Lipoma Diagnosed Incidentally on CT Scan and Its Review of Literature. J Clin Case Rep 3: 305.

2. Rozin AP, Toledano K (2013) New Sinusitis Associated Syndrome with Psoriasis and Periophthalmitis. J Clin Case Rep 3: 306

3. Shigefuku R, Takahashi $\mathrm{H}$, Ikeda $\mathrm{H}$, Matsunaga K, Koike J, et al. (2013) Case of Nonalcoholic Steatohepatitis Occurring in Combination with Sleep Apnea Syndrome, Who Showed Improvement of Liver Function by Continuous Positive Airway Pressure. J Clin Case Rep 3: 307.

4. Grover S, Somaiya M, Dutta P (2013) Use of Milnacipran in a Patient with Hyponatremia under the Cover of Fludrocortisones. J Clin Case Rep 3: 308.

5. Alibek Kenneth B, Ponomarev Igor A, Myrzaliyeva Dana B, Aituov Bauyrzhan A, Zhussupbekova Samal K, et al. (2013) A Case of Inflammatory-Edematous Breast Cancer Treated with Combination Therapy. J Clin Case Rep 3: 309

6. Docx MKF, Simons A, Ramet J, Mertens L,Govaert P (2013) Food Refusal, Loss of Appetite, Chronic Fatigue and Depression due to Central Adrenal Insufficiency Presenting as Anorexia Nervosa in an Adolescent Girl. J Clin Case Rep 3: 310.

7. Saber O, Gamal HE, Beevi F, Habeeb K (2013) A Rare Laryngeal Trauma: Complete Cricolaryngeal Separation. J Clin Case Rep 3: 311.
*Corresponding author: Dieudonne Kaimbo Wa Kaimbo, Ophthalmology and Biophysics, University of Kinshasa, Republic of Congo, E-mail: dieudonne_kaimbo@yahoo.com

Received December 29, 2013; Accepted December 31, 2013; Published January 01,2014

Citation: Wa Kaimbo DK (2014) Case Reports Covering Diagnostic, Pathogenic and Therapeutic Aspects of Diseases. J Clin Case Rep 4: e123. doi:10.4172/21657920.1000e123

Copyright: @ 2014 Wa Kaimbo DK. This is an open-access article distributed under the terms of the Creative Commons Attribution License, which permits unrestricted use, distribution, and reproduction in any medium, provided the original author and source are credited. 